\title{
Interactive Building
}

\author{
Henriette Bier \\ Faculty of Architecture, Delft University of Technology, Delft, The Netherlands \\ Email: h.h.bier@tudelft.nl
}

Received June 11, 2012; revised July 26, 2012; accepted August 10, 2012

\begin{abstract}
Distributed, networked, electronically tagged, interactive devices are increasingly incorporated into the physical environment blurring progressively the boundary between physical and virtual space. This changing relationship between physical and virtual implies not only a change in the operation and use of buildings but also a change in their physical configuration, and therefore, their design and production. Interactive building addresses, therefore both the building defined as physically built environment and the building process implying on the one hand the changing role of architecture with respect to incorporation of interactivity and the resulting multiple and varied use of built environments in reduced timeframes; On the other hand, it is implying the changing role of the architect with respect to the use of networks connecting digital databases and parametric models with customizable design and production tools allowing for linking design to production and use. This paper discusses both by presenting two case studies within the larger framework of interactive building.
\end{abstract}

Keywords: Interactive Built Environments; Interactive Design-Production-Use; Participatory Design; Re-Configurable Architecture

\section{Introduction}

The progressive integration of networked, interactive devices into the physical environment is implying a transformation not only in the operation and use of built environments but also a change in their physical configuration, and therefore, their design. While physically built environments are increasingly incorporating aspects of ambient intelligence [1,2] employing data contained within the network connecting them [3], the relevant question becomes not whether intelligent, sentient environments may be built, but how these environments may become instrumental in distributed problem solving [4] and how (artificial) intelligence may be embedded into buildings in order to serve everyday activities [5].

This paper addresses, therefore, the changing role of the architectural project and the changing role of the architect in the process of progressive incorporation of distributed, networked, interactive devices into the built environment.

\section{Interactive Built Environments}

Considering the Internet as the start, Castells [6] extrapolated 1996 the expected future development of such a networked system towards becoming pervasive, permeating every-day life activities. The purpose of Internet-supported systems is, indeed, not anymore to exclusively bridge time and space but to support (even replace) every-day activities such as banking, shopping, learning, etc. and, therefore, augment even radically change relevance, meaning, and use of physically built space.

While both, the physical and the virtual facilitate the social, the question is how do they relate to each other and how do they influence architecture? What does architecture become when physical interaction is increasingly complemented or even replaced by virtual interaction? How may built environment change when it incurporates aspects of ambient intelligence?

In a case study, the 24/7 use of the main market place in Rotterdam was investigated with a group of students (http://www.indesem.nl/) during a one-week workshop. The 24/7 use was digitally mapped in form of data fields showing time-based increase and decrease of activities and use, whereas two relevant conditions were identified: Market and no-market days would imply a drastic change from high-to low-density use (Figure 1) requiring two radically different spatial configurations. The design proposal addressed these conditions by introducing a voxelized, interactive landscape (Figure 2) that would reconfigure spatially for market and no-market days, accordingly, whereas the no-market configuration was developed into more detail. While the configuration for market days requested stable rows of tables between walk paths, the configuration for no-market days implied the slow but constant change of the landscape (Figure 2) based on the principle that walking and bicycle paths or 

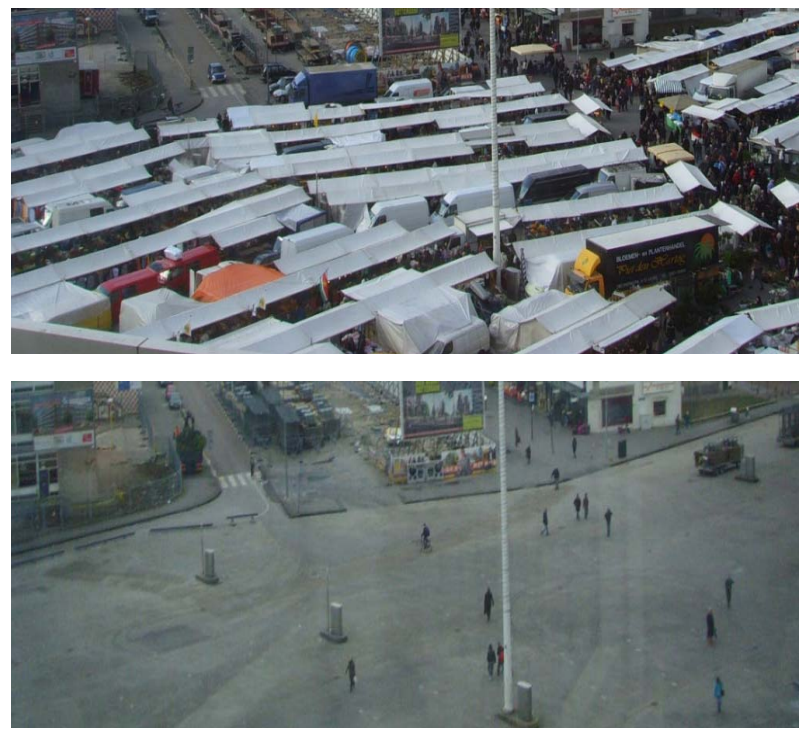

Figure 1. Market (above) and no-market (below) activity mapping shows fluctuation of use.
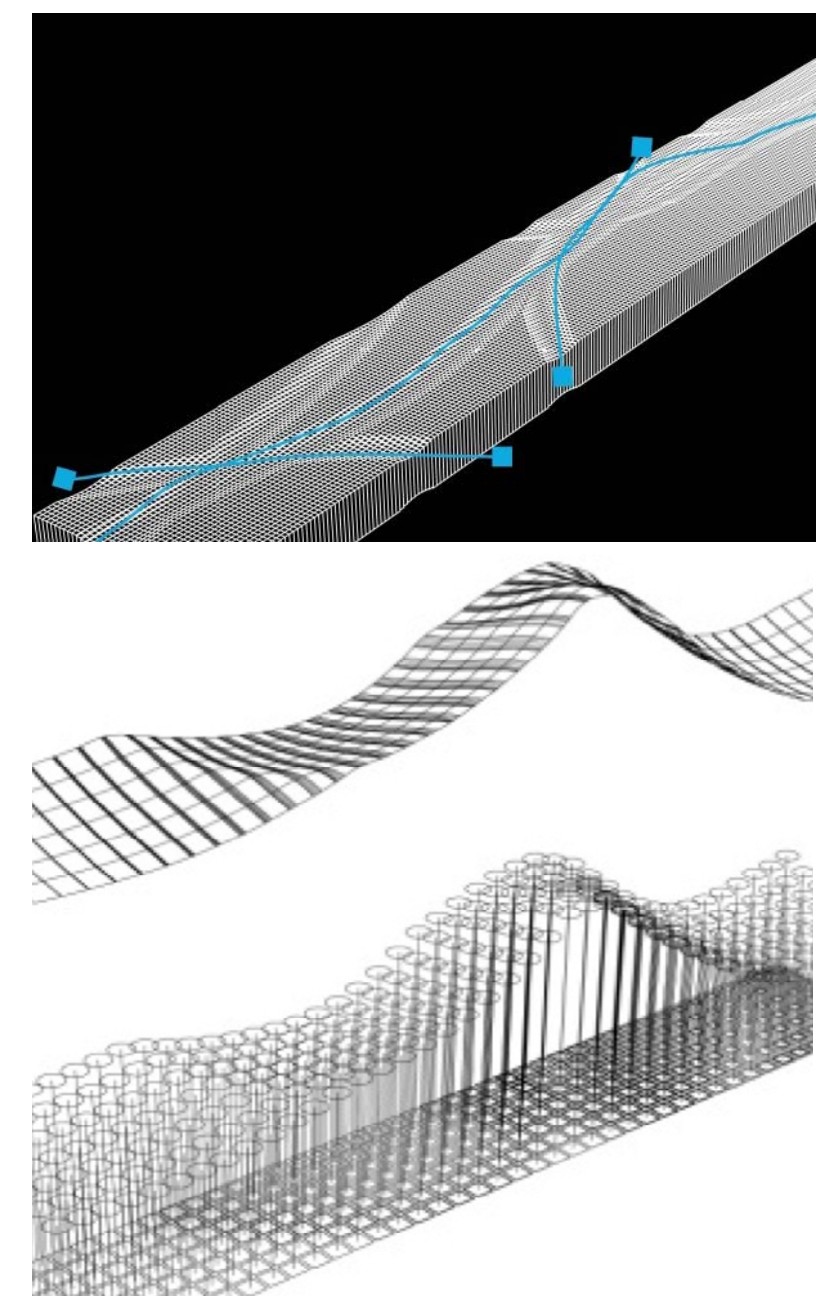

Figure 2. Reconfigurable (voxelized) interactive landscape is adapting to $24 / 7$ use of market place whereas paths form only when they are activated due to use. seating areas would form only when they would be activated due to use (Figure 3).

With respect to technical implementation such a system is envisioned to consist of individual 50/50/50 cm voxels covering the whole street surface. The voxels are operated from below ground by electronic cylinders (pistons) driven by servomotors, whereas each element can move independently. Each voxel has a motor controller, which directly controls the position of the servomotors, and therefore, the pistons.

Communication and control between voxels and users may be implemented similarly to other Hyperbody interactive projects such as the InteractiveWall [7] by employing customized circuits built around Arduino, which is an open-source electronics prototyping platform. For sensing, motion sensors capable of detecting distance may be employed, whereas each voxel may have at least one sensor at the topside of the voxel. Via software such as Max/MSP/Jitter sensors may be combined to create a representation of the complete sensor space, which may be used to interpret user presence. The interface may allow monitoring, calibrating and filtering sensors so that local and global behavior of the system may be controlled. Local behavior may address simple rules such as: 1) Move up when movement stagnates; 2) Move down when movement intensifies; and 3) When no movement detected mediate position between neighbors. Whereas global control may initiate, dim or stop interaction.

Via embedded sensors voxels may be responsive to approaching users and they often may need to negotiate between fast, slow moving, and not moving users. This is resolved by addressing only conditions of stagnation or movement that take place (depending on speed) longer than 5 - 10 minutes.

This case study aims to prove that interactive, reconfigurable architecture incorporating ambient intelligence allows for multiple use of public spaces in condensed timeframes, while virtual and physical borders start to blur in order to facilitate social interaction. Such reconfigurable, interactive architecture addresses, therefore, society relevant issues such as efficient use of space, user-inspired design, varied and multiple use of space, whereas digital mapping, modeling, and manufacturing establish a direct link between design conceptualization, process, result and use.

This implies that interactive, context aware (sensoractuator) sub-systems are embedded into the spatial environment in such a way that they are context and user aware by collecting and mapping data with respect to users' movement and behavior in relation to physical space, they are even tailored to individual needs, and furthermore, they are adaptive, responding to user and environmental changes, even anticipatory, as for instance described in following sections. Considering that informa- 


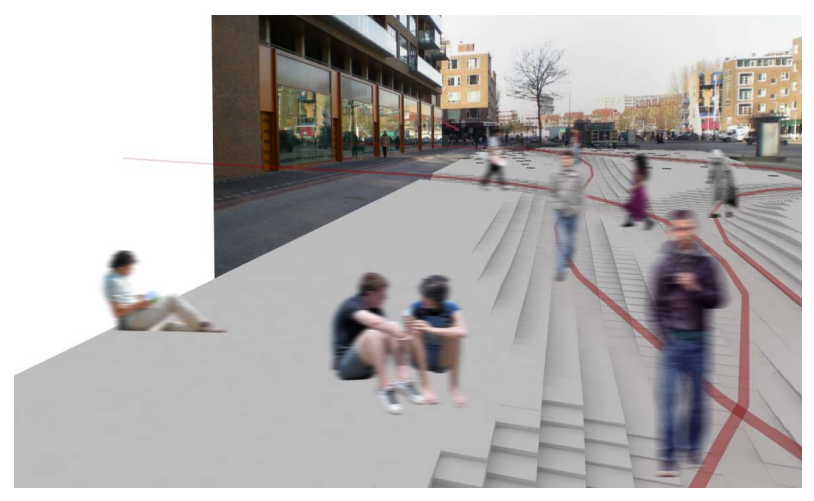

Figure 3. Paths and surfaces to sit on form only when they are activated by use.

tion processing has been, meanwhile, increasingly integrated into physical spaces i.e. architecture, everyday objects, and human activities [8] and ubiquitous computing has become prevalent in everyday life, the following section aims to critically asses what they offer architectural production, and thus reveal what challenges remain in their development and application.

\section{Interactive Building}

Paraphrasing Benjamin's statement [9] from 1936 that mechanical reproduction has freed the work of art from its dependence on ritual, which implied a move from drawing towards photography and film, digital production (and reproduction) today's development towards animation and simulation is addressing principles [10] such as Numerical Representation (code), Assemblage (of multimedia compounds), Multiplicity (of versions of media assemblies), Interaction, Automation, and Transcoding (as a process of translation and conversion from one digital medium to another).

Applied to architectural design and its representation as well as its production these principles imply that: Representation of design is, inter alia, analog and digital; figurative (metaphorical) and abstract; 2 - 4 dimensional and interactive; codified, diagrammatic, schematic and descriptive, detailed, comprehensive; Furthermore, production (materialization) is directly linked to such 2 - 4 dimensional representations of (architectural) design via Computer Numerically-Controlled (CNC) machines enabling:

1) Generation of mass-customized and individualized designs by incorporating the user in a participatory design process where the architect develops customizable meta-designs and

2) Use of networks connecting digital databases and parametric models with customizable design and production tools allowing for directly linking design to production and use.

\subsection{Interactive Design and Fabrication}

Parametric, digitally-driven design and fabrication frees architectural form from constraints imposed by industrial production based on repetition and standardization: Architecture becomes mass-customized connecting directly parametric design with physical production via CNC fabrication, enabling, on the one hand, design and production of unique designs, on the other hand, enabling design and production of individualized designs by incorporating the user in a participatory design process where the architect develops customizable meta-designs. In this context, meta-design refers to a framework within which variable and fixed-parameters are defined in such a way that customization is, while constrained, possible. In principle, meta-design is implemented while acknowledging that future uses (and potential problems) cannot be exhaustively investigated and anticipated at the time of the design. This implies the need of integrating change at the design, production and use level.

Interactive design and fabrication has been explored at Hyperbody in Protospace, which is an Internet-based, multi-user environment enabling real-time collaborative architectural and urban design. Protospace is, basically, a compound of software and hardware applications for academic education and research (Figure 4) equipped with multi-channel immersive audio, multi-screen projection, ubiq-uitous sensing, wireless input-output devices and CNC facilities (incorporating laser cutter and milling machine). Protospace facilitates Internet-supported collaborative and participatory design and production of interactive architectural components, in a process of seamless Computer-Aided Design and Manufacturing (CAD \& M) workflows, as well as implementation of non-standard, complex geometries into architecture.

Protospace incorporates, therefore, virtual drafting and modeling as well as physical prototyping tools with shared database capabilities so that changes made by design team members are visible to all other team members, thus allowing for design, evaluation, and dialogue between team members to take place concurrently in real-time. Team members (users) are physically and/or virtually participating in a seamless process of designing, engineering, prototyping, and reviewing, and therefore, establishing a feedback loop between conceptualization and production.

In such a networked system, users are connected with other users, multimedia databases, parametric models, and software-hardware applications enabling reading and editing of data, sensing-actuating, and computing in such a way that users interact physically and virtually as needed in this physical, digitally-augmented environment. By integrating concepts such as Autonomous Control [11] Protospace operates within the Internet of Things as a system in which self-organized virtual and physical 


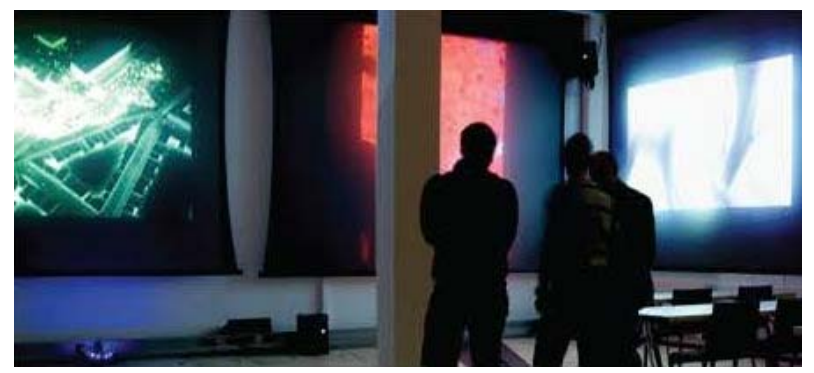

Figure 4. Protospace real-time collaborative architectural and urban design.

agents such as users, data, and equipment are able to act and interact autonomously with respect to context and environmental factors. Such context awareness [12] implies data collection and information exchange thus communication between users and physical environment implying acquisition of data with respect to users' habits, bodily states, their interaction, and their regular and spontaneous activities as well as context data with respect to spatial location, infrastructure, available resources, and physical conditions such as noise, light, and temperature. Information exchange thus communication between physical (sentient) environment and users may, however, not only imply accommodating but also challenging interactions.

As a context aware system, Protospace is concerned with the acquisition of context data by means of sensors, as mentioned before, the interpretation of the data collected by sensors, and the triggering of accommodating and challenging actions as response to the interpretation of collected data, whereas responses may imply operation of electrical light, sun shading, and projection screens, depending on local and global requirements, etc. Furthermore, Protospace's context awareness addresses, also, activity recognition as implemented in interactive lectures and $\mathrm{CAD} \& \mathrm{M}$ sessions.

\subsection{Interactive Operation}

Distributed, networked, interactive devices linking digital databases and parametric models with customizable design and production tools allow for distributed participatory building and operation. In this context, BIM (Building Information Modeling) connects not only design with physical production but also with cost, construction, and project management as well as (postproduction) building operation. This implies sharing of digital databases, libraries, and parametric models among involved experts (architect, civil, structural, construction engineer, etc.) and the end-user (operator).

BIM therefore incorporates, in addition to geometry, spatial relationships, light and environmental conditions, demographic and geographic information, quantities, qualities and properties of building components, etc. It, basically, represents the design as a set of parametric characteristics and relations-vague and defined, generic and product-specific, material and immaterial-enabling active involvement of all stakeholders (experts, customers, partners, end-users) in the design and production process in order to ensure that the product meets all requirements, the built environments are responsive to user-specific needs and appropriate with respect to formal, functional, structural, environmental demands.

\section{Conclusions}

Due to the shift from mechanical, standardized industrial production (Modernism, CIAM/Functionalism, etc.) to digital mass-customization and the shift from inanimate (inert, insentient) towards animate (actuated, sentient) architectural environments [13] research into interactive building has become increasingly relevant because of its effect on societal issues such as inefficient use of resources and built space. The society seems, therefore, to move from a consumer-towards a producer-oriented and participatory social and material interaction [14].

In this context, interactivity is addressed not only at the object level where building components and buildings become dynamic, acting and re-acting in response to environmental and user-specific needs, but also at the process level, where the design and production of building components and buildings is participatory and interactive in nature.

\section{Acknowledgements}

This paper has benefited from the contribution of Hyperbody, Protospace, InDeSem (2011), and Border Conditions research and design teams.

\section{REFERENCES}

[1] E. Aarts and S. Marzano, Eds., "The New EverydayViews on Ambient Intelligence,” 010 Publishers, Rotterdam, 2003.

[2] T. Gross, "Towards a New Human-Centred Computing Methodology for Cooperative Ambient Intelligence,” Journal of Ambient Intelligence and Humanized Computing, Vol. 1, No. 1, 2010, pp. 31-42.

[3] K. Ashton, “That 'Internet of Things' Thing," Radio Frequency Identification Journal, 2009. http://www.rfidjournal.com/article/view/4986

[4] M. Novak, "Cognitive Cities: Intelligence, Environment and Space," Intelligent Environments-Spatial Aspect of the Information Revolution, Elsevier, Oxford, 1997, pp. 386-420.

[5] E. Aarts, R. Harwig and M. Schuurmans, "Ambient Intelligence,” The Invisible Future: The Seamless Integration of Technology Into Everyday Life, McGraw-Hill Companies, New York, 2001, pp. 235-250. 
[6] M. Hosale and C. Kievid, "Modulating Territories, Penetrating Boundaries," Digitally-Driven Architecture, 6th Footprint Issue, Stichting Footprint, Delft, 2010, pp. 5568.

[7] M. Castells, “The Rise of the Network Society,” The Information Age: Economy, Society and Culture, Vol. 1, Blackwell Publishers, Malden, 1996.

[8] D. Russell and M. Weiser, "The Future of Integrated Design of Ubiquitous Computing in Combined Real and Virtual Worlds,” Proceedings of CHI-98, Los Angeles, 18-23 April 1998, pp. 275-276.

[9] W. Benjamin, "Das Kunstwerk im Zeitalter Seiner Technischen Reproduzierbarkeit-Essenzen,” Suhrkamp Verlag, Berlin, 2006.

[10] L. Manovich, “The Language of New Media,” Massachusetts Institute of Technology Press, Cambridge, 2001.
[11] D. Uckelmann, et al., “Autonomous Control and the Internet of Things: Increasing Robustness, Scalability and Agility in Logistic Networks,” Unique Radio Innovation for the 21st Century: Building Scalable and Global RFID Networks, Springer, Berlin, 2010, pp. 163-181. doi:10.1007/978-3-642-03462-6_8

[12] H. Gellersen, M. Beigl and A. Schmidt, "Sensor-Based Context-Awareness for Situated Computing," Workshop on Software Engineering and Pervasive Computing at ICSE 2000, Limerick, 2000.

[13] H. Bier and T. Knight, "Digitally-Driven Architecture," In: H. Bier and T. Knight, Eds., 6th Footprint Issue, Stichting Footprint, Delft, 2010, pp. 1-4.

[14] D. Uckelmann1, M. Harrison and F. Michahelles, “An Architectural Approach towards the Future Internet of Things," Architecting the Internet of Things, Springer, Berlin, 2011, pp. 1-24. 